\section{Efficacy and Safety of Nemolizumab for Treatment of Adult Atopic Dermatitis}

Freemantle $\mathrm{N}^{1}$, Piketty $\mathrm{C}^{2}$

${ }^{1}$ Institute of Clinical Trials and Methodology, University College

London, London, UK

${ }^{2}$ Galderma, Lausanne, Switzerland

J Investig Allergol Clin Immunol 2021; Vol. 31(6): 528-529 doi: $10.18176 /$ jiaci.0727

Key words: Nemolizumab. Adult atopic dermatitis.

Palabras clave: Nemolizumab. Dermatitis atópica del adulto.

\section{To the Editor:}

We were interested to read the meta-analysis by Xuemin et al [1] on the efficacy and safety of nemolizumab for treatment of adult atopic dermatitis. This is an important area, and an overview of the available data is timely. We concur with the conclusions of the authors that nemolizumab is a promising treatment, and Galderma is currently undertaking further phase 3 clinical trials to gather new data.

On one important point, however, we struggle to reconcile the results and conclusions of the review with our knowledge of the published data. The authors comment that the $60-\mathrm{mg}$ Q4w regimen is likely to be optimal. The only complete phase 3 trial that has compared that dose [2] on a proper like-

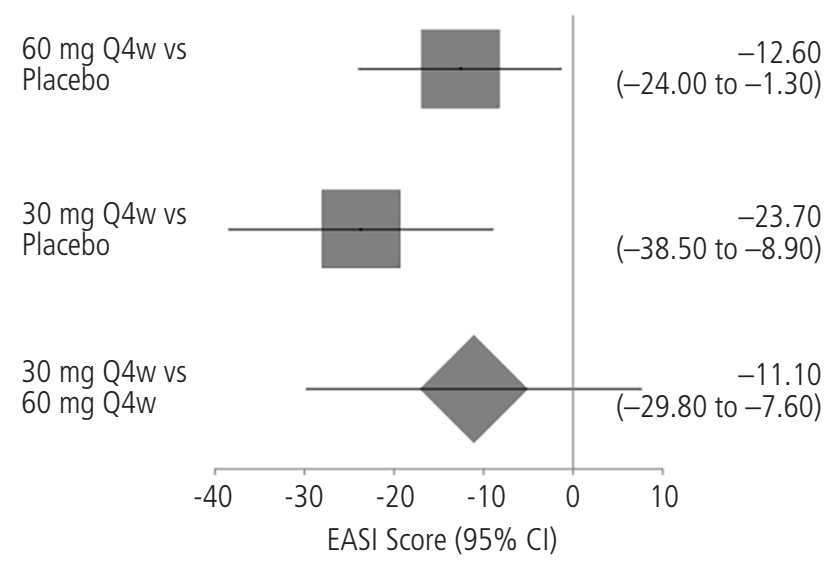

Figure. Percent change in Eczema Area and Severity Index (from baseline to week 16) by dose.

The 60-mg Q4w vs placebo comparison is derived from the phase 3a trial of Kabashima et al [2]; the 30-mg Q4w versus vs comparison is derived from the phase 2 trial of Silverberg et al [3]. The difference between doses is calculated using the method of Bucher et al [4]. for-like basis with a lower dose currently in late development (30 mg Q4w) is actually in favor of the lower dose. The percent change in the Eczema Area and Severity Index (EASI) (from baseline to week 16) for the 30-mg Q4w dose vs placebo (difference, $-23.7 ; 95 \% \mathrm{CI},-8.9$ to $-38.5 ; P=.002$ ) was reported in the phase $2 b$ trial of Silverberg et al [3]. The treatment effect observed with EASI was greater than that observed with the $60-\mathrm{mg}$ Q4w dose in the phase 3 trial by Kabashima et al [2] (difference, $-12.6 ; 95 \% \mathrm{CI},-1.3$ to -24.0 ). Using the method of Bucher et al [4] to undertake a formal indirect comparison, we observed a numerically greater benefit for $30 \mathrm{mg}$ Q4w (difference, -11.1 ; 95\% CI, 7.6 to -29.8) (Figure). The 30-mg dose is under evaluation in 2 pivotal phase 3 trials (NCT03989349 and NCT03985943), and analysis of the outcomes of both will help us resolve this important question.

We do not recognize some of the data included by the reviewers in their work; for example, the results above for Silverberg et al [3] derived from the clinical study report are not reflected in the report by Xuemin et al [1]. This is troubling. While the overall conclusions are supported by individual trials, the dosage conclusions do not reflect current data.

\section{Funding}

This work was funded by Galderma.

\section{Conflicts of Interest}

NF has received consulting fees from ALK Allergan, Aimmune, AstraZeneca, MSD, Ipsen, Sanofi Aventis, Novo Nordisk, Novartis, and Grifols and speaking fees from Abbott Singapore and Sanofi Aventis. His institution receives a grant from the European Association for Cardiothoracic Surgery for methodological advice and teaching. CP is an employee of Galderma.

\section{References}

1. Xuemin $X$, Lihang $L$, Changhua Z, Xiaofang $Y$, Yongshan $N$, Zhipeng $L$, et al. Efficacy and safety of nemolizumab for adult atopic dermatitis treatment: A meta-analysis of randomized clinical trials. J Investig Allergol Clin Immunol. 2021;31:1902.

2. Kabashima K, Matsumura T, Komazaki H, Kawashima M, and the Nemolizumab-JP01 Study Group. Trial of Nemolizumab and Topical Agents for Atopic Dermatitis with Pruritus. N Engl J Med. 2020;383:141-50.

3. Silverberg JI, Pinter A, Pulka G, Poulin Y, Bouaziz JD, Wollenberg $A$, et al. Phase $2 B$ randomized study of nemolizumab in adults with moderate-to-severe atopic dermatitis and severe pruritus. J Allergy Clin Immunol. 2020;145:173-82. 
4. Bucher HC, Guyatt GH, Griffith LE, Walter SD. The results of direct and indirect treatment comparisons in meta-analysis of randomized controlled trials. J Clin Epidemiol. 1997;50:68391.

- Manuscript received May 28, 2021; accepted for publication June 25, 2021.

\section{Nick Freemantle}

Professor of Clinical Epidemiology \& Biostatistics Institute of Clinical Trials and Methodology

University College London

90 High Holborn, 2nd Floor

London WC1V 6LJ

UK

E-mail: nicholas.freemantle@ucl.ac.uk 